Original Paper http://ajol.info/index.php/ijbcs http://indexmedicus.afro.who.int

\title{
Evolution de la diversité des cultivars de fonio pratiqués dans la commune de Boukoumbé au Nord-Ouest du Bénin
}

\author{
Emmanuel SEKLOKA ${ }^{1 *}$, Hubert ADOUKONOU-SAGBADJA ${ }^{2}$, Armand A. PARAÏSO ${ }^{3}$, \\ Brigitte KOUEGA YOA ${ }^{1}$, François-Xavier BACHABI ${ }^{4}$ et \\ Nouhoum ZOUMAROU-WALLIS ${ }^{1}$
}

\author{
${ }^{1}$ Université de Parakou, Faculté d'Agronomie, Département de Production Végétale, Laboratoire de \\ Phytotechnie, d'Amélioration et de Protection des Plantes, BP 123 Parakou, République du Bénin. \\ ${ }^{2}$ Université d'Abomey-Calavi, Faculté des Sciences et Techniques, Laboratoire de Biotechnologie, \\ BP : 01 BP 526 Cotonou, République du Bénin. \\ ${ }^{3}$ Université de Parakou, Faculté d'Agronomie, Département de Production Végétale, \\ Laboratoire de Protection des Végétaux, de Parasitologie et de Pathologie des Abeilles, \\ BP 123 Parakou, République du Bénin. \\ ${ }^{4}$ Université de Natitingou, Ecole Nationale des Sciences et Techniques Agricoles, \\ Département de Production Végétale, BP 73 Djougou, République du Bénin. \\ *Corresponding author; E-mail: emmanuelsekloka@hotmail.com
}

\section{REMERCIEMENTS}

Ce travail a été sponsorisé par le Conseil Scientifique de l'Université de Parakou à qui nous adressons nos sincères remerciements.

\section{RESUME}

Afin de comparer la diversité actuelle des écotypes de fonio à celle cultivée dix (10) ans plus tôt au Bénin, une enquête a été menée en 2013 dans la commune de Boukoumbé. Dans chacun des vingt (20) villages prospectés, un focus groupe d'une douzaine de producteurs a été à chaque fois enquêté. Cinq (5) à dix (10) producteurs sont ensuite entretenus individuellement à l'aide d'un questionnaire semi-structuré pour recueillir des informations sur les cultivars, leurs noms locaux, leurs principales caractéristiques, et la gestion des semences. Les résultats ont confirmé la diversité des appellations et ont permis de recenser cinq (5) cultivars différenciés par la précocité, la couleur des racèmes, les tailles de graines et de plantes. Les semences, transmises surtout de génération en génération, sont prélevées à $99 \%$ sur les récoltes précédentes. Près de $95 \%$ des enquêtés cultivent une seule variété à la fois. La plus forte diversité a été rencontrée chez les "Ditamaris" des villages de Boukoumbé-centre et Natta. "Iporapia" et "Takotapuaka" sont cultivés par toutes les ethnies. La diversité décrite dix (10) ans plus tôt dans la zone a été maintenue. Toutefois, les variétés de précocités extrêmes sont de moins en moins cultivées, et donc menacées de disparition.

(C) 2015 International Formulae Group. All rights reserved.

Mots clés : Espèce négligée, accession de fonio, Digitaliaspp., conservation in situ, amélioration variétale, Bénin. 


\title{
Evolution of the diversity of fonio cultivars practiced in the commune of Boukoumbe in North-West of Benin
}

\begin{abstract}
To compare the current diversity of landraces of millet fonio to that cultivated ten (10) years ago in Benin, a survey was conducted in 2013 in the district of Boukoumbé. In each of the twenty (20) villages surveyed a focus group of a dozen producers was investigated every time. Then, five (5) to ten (10) producers are individually interviewed using a semi-structured questionnaire to collect information on cultivars, their local names, their main characteristics and seed management. The results confirmed the diversity of names and allowed to identify five cultivars differentiated by the earliness, color of racemes, sizes of seeds and plant height. Seeds, especially transmitted from generation to generation, are taken at $99 \%$ on the previous harvest. Nearly $95 \%$ of respondents grow only one variety at a time. The greatest diversity was encountered among the "Ditamaris" of villages in Boukoumbé center and Natta. "Iporapia" and "Takotapuaka" are cultivated by all ethnic groups. The diversity described ten (10) years earlier in the area has been maintained. However, the extreme precocity varieties are lesser and lesser grown, and therefore at risk of extinction.
\end{abstract}

(C) 2015 International Formulae Group. All rights reserved.

Keywords: Neglected species, accession of fonio, Digitaria spp, in situ conservation, plant breeding, Benin.

\section{INTRODUCTION}

Plus de 7000 plantes sont cultivées ou collectées à l'état sauvage à travers le monde pour l'alimentation (CIRAD, 2010). De plus, il est estimé que près de trois-quarts de la diversité génétique des cultures agricoles ont été perdus au cours du dernier siècle, et cette érosion génétique se poursuit (Olivieri et Vitalis 2001 ; CIRAD 2010). Le blé, le riz et le maïs à eux seuls fournissent plus de $50 \%$ de l'apport énergétique à base de plantes (CIRAD, 2010). Cet état de fait provient d'orientations fortes en matière de politique, de recherche et d'amélioration des plantes qui ont focalisé toutes les énergies sur des espèces qualifiées de "majeures".

Un grand nombre d'espèces ont été négligées (pas ou peu de recherches ou d'actions de promotion) ou sous-utilisées (valeur potentielle sous-estimée ou sousexploitée). Or, ces espèces présentent des caractéristiques intéressantes dans un contexte de croissance démographique et de changements climatiques (CIRAD, 2010). Elles sont produites localement (bonne adaptation à leur zone de culture), elles contribuent à la sécurité alimentaire, sont facilement voire gratuitement accessibles (semences familiales, plantes sauvages) aux populations, peuvent même être une importante source de revenus (Paraïso et al., 2011), et enfin, ont une bonne résilience à la fois d'un point de vue climatique que d'un point de vue conservation dans des zones marginales.

Le fonio blanc (Digitariaexilis) est l'une d'elles. C'est une céréale cultivée en Afrique de l'Ouest, dans une région s'étendant du Sénégal au lac Tchad (Cruz et Béavogui, 2011). Elle est considérée comme la plus ancienne céréale d'Afrique occidentale.

La culture $\mathrm{du}$ fonio est faite annuellement sur près de 450000 ha et la production, voisine de 360000 tonnes (rendement moyen de $800 \mathrm{~kg} / \mathrm{ha}$ ) assure l'alimentation de plusieurs millions d'êtres humains durant les mois les plus difficiles au point de vue des ressources alimentaires (Cruz et Béavogui, 2011).

Au Bénin, la principale zone de culture du fonio est la région de Boukoumbé et ses environs. Cette céréale y joue un rôle 
socioculturel et économique très important (Paraïso et al., 2011; Ballogoun, 2012) et contribue à l'alimentation des populations en période de soudure. Malheureusement, elle bénéficie de très peu d'attention de la part de la recherche. En conséquence, les variétés cultivées sont toujours traditionnelles et leur production est en déclin (Dansi et al., 2010 ; Paraïso et al., 2011). Dans cette localité qui est une des zones d'origine de cette céréale en Afrique (Adoukonou-Sagbadja et al., 2007), la multiplicité des appellations de fonio complique les travaux de recensement et de caractérisation des cultivars. De plus, comme dans la plupart des régions d'Afrique de l'Ouest où cette céréale est cultivée, il n'existe pas, pour cette spéculation, de système formel de création de variété, de production et de distribution de semences. L'accès aux semences est essentiellement traditionnel, basé majoritairement sur le prélèvement de semence sur la récolte de l'année précédente, sur des échanges de semences entre agriculteurs et quelques rares fois sur achat de semences au marché (Adoukonou-Sagbadja et al., 2006, Dansi et al., 2010). Dans ces conditions, la diversité variétale et son évolution dans le temps sont assez souvent difficiles à appréhender. Un premier travail d'inventaire de cultivars de fonio, réalisé en 2003 dans la zone de Boukoumbé et ses environs, a permis de caractériser cinq morphotypes dont quatre seraient de Digitaliaexilis et un de D. iburua (Adoukonou-Sagbadja et al., 2007 ; Dansi et al., 2010). Compte tenu du mode traditionnel d'accès aux semences et de l'inexistence d'un système formel de conservation de ces ressources génétiques, on pourrait se demander, dix ans après, si cette diversité variétale a été conservée ou non. Le présent travail s'inscrit dans ce cadre et vise à clarifier l'état de la diversité et la complexité des appellations de cultivars de fonio à Boukoumbé d'une part, et de l'autre, à apprécier l'évolution de la diversité variétale de cette espèce dans la zone d'étude.

\section{MATERIEL ET METHODES Site de l'étude}

L'étude a été conduite en 2013 dans la commune de Boukoumbé au Nord-Ouest du Bénin, dans le département de l'Atacora, situé entre $10^{\circ}$ à $10^{\circ} 40^{\prime}$ de latitude Nord et $0^{\circ} 50^{\prime}$ à $1^{\circ} 30^{\prime}$ de longitude Est. Elle est limitée au Nord-Est par la commune de Tanguiéta, au Nord-Ouest par celle de Cobly, au Sud par la commune de Natitingou, à l'Est par la commune de Toucountouna et bordée par la chaîne de l'Atacora et à l'Ouest par la République du Togo (Figure 1).

\section{Méthodologie}

Les enquêtes ont été menées dans les sept (07) arrondissements de la commune, à savoir: Boukoumbé-Centre, Korrontière, Manta, Natta, Dipoli, Koussoucoingou et Tabota. Tous les arrondissements ont été sélectionnés afin de couvrir la diversité des cultivars et de leurs appellations locales dans la commune, étant donné que Boukoumbé fait partie de la zone d'origine des variétés de fonio cultivé en Afrique de l'Ouest (Adoukonou-Sagbadja et al., 2007). Dans chacun de ces arrondissements, deux à trois villages réputés dans la culture du fonio ont été sélectionnés. Dans chaque village, un focus groupe, composé d'une douzaine de producteurs de fonio, a été enquêté. Au cours de l'entretien avec ces focus groupes, les objectifs de l'étude sont présentés et les discussions ont permis à chaque fois d'adapter un questionnaire préalablement conçu (Ballogoun, 2013), et de recueillir les premières informations sur la connaissance des différentes variétés cultivées. Après chaque discussion de groupe, cinq (5) à dix (10) producteurs de fonio étaient choisis parmi les plus expérimentés pour des entretiens individuels. Au total, cent quarante 
(140) producteurs individuels de fonio répartis dans vingt (20) villages (Tableau 1) ont été ainsi enquêtés à l'aide d'un questionnaire semi-structuré.

Les données recueillies ont permis de rendre compte des différentes variétés de fonio cultivées par les enquêtés, leurs noms locaux et leurs principales caractéristiques morphologiques et agronomiques. Ces données ont été validées à l'aide de la méthode de triangulation des informations (Apostolidis, 2005). Dans chaque village, les autorités locales (chef du village, chefs coutumiers, etc.) et les agents de vulgarisation ont été impliqués afin de faciliter l'organisation des discussions de groupe avec les producteurs. Les données recueillies ont été ensuite dépouillées et analysées avec le logiciel Excel à l'aide des outils de la statistique descriptive.

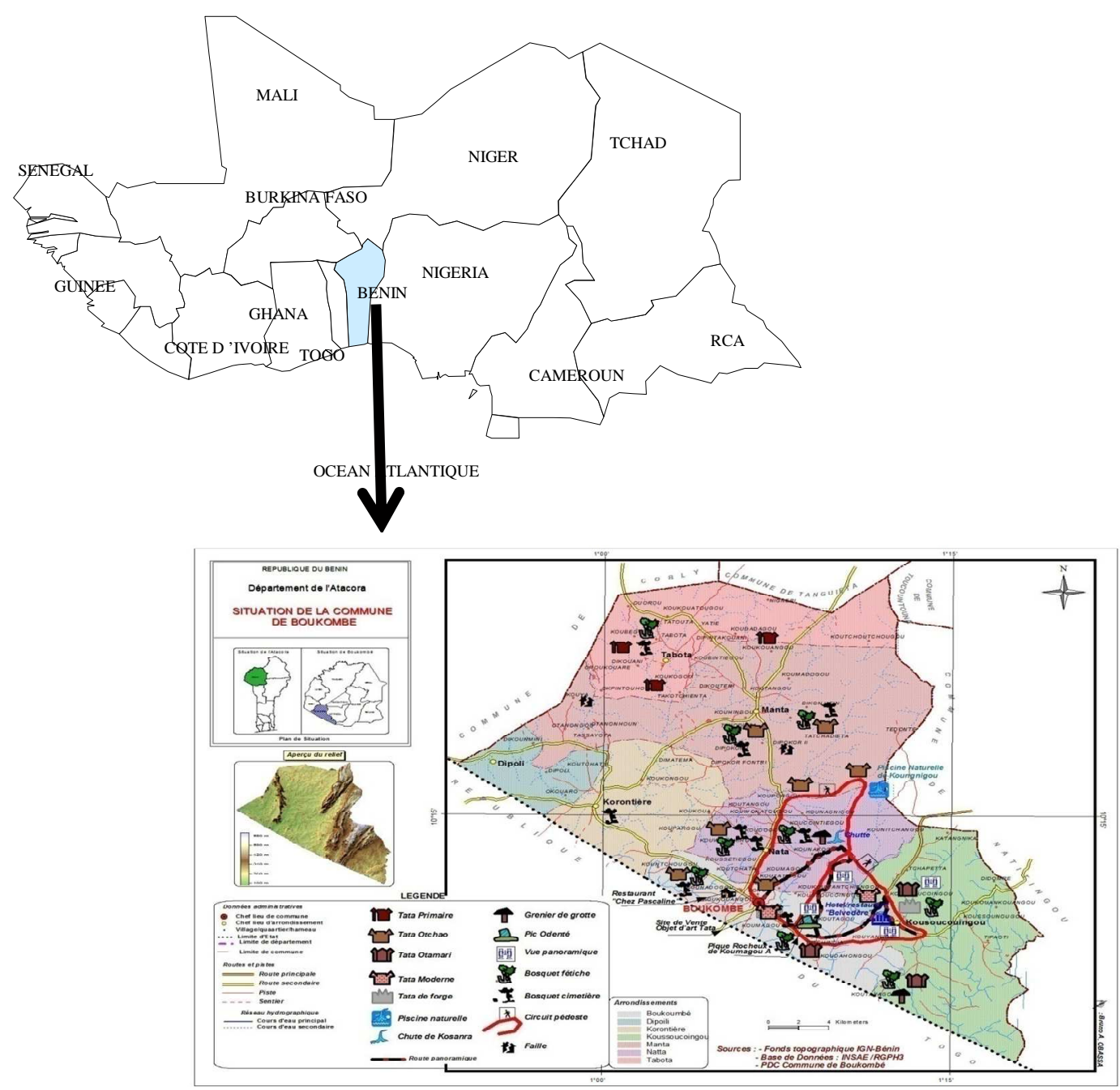

Figure 1 : Localisation géographique de la zone de l'étude. 


\section{RESULTATS \\ Identification des producteurs de fonio enquêtés}

A Boukoumbé, le fonio est essentiellement cultivé par les hommes (97\% des producteurs enquêtés). Le producteur le plus jeune avait dix-neuf (19) ans et le plus âgé avait soixante-seize (76) ans. La moyenne d'âge était de trente-huit (38) ans, avec le maximum de producteurs compris entre vingt (20) et cinquante (50) ans (83\%) (Figure 2). Tous les producteurs enquêtés étaient mariés. Un seul des producteurs enquêtés était chrétien, le reste pratiquait des religions endogènes (animistes). Les enquêtés étaient en majorité des analphabètes $(80 \%) ; 13 \%$ avaient le niveau primaire et $7 \%$ le niveau secondaire. La principale activité était l'agriculture et ils pratiquaient majoritairement l'élevage en activité secondaire. Quelques-uns associaient à l'élevage, la chasse et/ou la pêche. Deux des producteurs qui avaient un niveau secondaire exerçaient dans l'enseignement communautaire, l'un en activité principale, l'autre en activité secondaire.

Cultivars de fonio recensés à Boukoumbé pendant la période d'étude

A Boukoumbé les appellations des cultivars de fonio varient énormément suivant les groupes ethniques et parfois, pour un même groupe ethnique, suivant la situation géographique dans la commune. Par exemple, le cultivar appelé "yoro" en Lamba est appelé différemment par l'ethnie Ditamari (l'ethnie la plus répandue à Boukoumbé) selon la situation géographique dans la commune:

- "Dakotapora" dans le village de Mantchari (Dipoli) ;

- "Datépora"dans le village de Dissapoli (Dipoli) ;

• "Ikantoni", "Ikantoga" et "Kotapoada"dans le village de Yatier (Tabota) ;

- "Tintingoula"dans le village de Diporko-1 (Manta)

- "Datépoara" et "Kotapoaga" à Boukoumbé centre ;

- "Tententira" et "Dakotapora" dans le village de Koudorgou (Natta);

- "Takotapora" et "Datépora"dans le village de Tassayota (Korontière).

Certaines appellations résultent d'une abréviation du nom bien connu de la variété C'est le cas par exemple de "Ipia" et de "Ini" qui dérivent respectivement de "Iporapia" et de "Iporni" qui sont toutes utilisées indifféremment par les producteurs en même temps que ces abréviations. Pour d'autres, il s'agit simplement du nom de l'ethnie qui cultive originellement le cultivar. C'est le cas de "Iporapia", originellement cultivé par les Lamba, et qui est encore appelé "Namba" (déformation de Lamba) par les Ditamari du village Yatier dans l'arrondissement de Tabota. Le Tableau 2 présente la liste des différentes appellations des cultivars de fonio suivant les villages et suivant les ethnies rencontrées à Boukounbé.

A l'issue de la triangulation des informations collectées et en se basant sur les correspondances entre les noms des variétés, correspondances discutées et établies avec les producteurs expérimentés dans les différents arrondissements, les appellations correspondent en définitive à cinq différents cultivars de fonio (Tableau 3) : "Iporapia" qui signifie fonio blanc, "Iporwan" (fonio rouge), "Takotapuaka" (fonio de poule), "Ipuaka-ini" (fonio femelle) et "Ipuaka Ida" (fonio mâle).La plus grande diversité d'appellations a été rencontrée chez les Ditamari où chaque variété a au minimum sept (7) noms suivant la situation géographique dans la commune. Chez les Lamba par contre, les appellations des cultivars sont beaucoup moins diversifiées (au plus 4 par cultivar). Le cultivar Takotapuaka est celui qui a moins d'appellations : sept (7) en Ditamari, un (1) en M'Bermè et un (1) en Lamba (Tableau 3).

\section{Description des cultivars identifiés}

Les principaux critères de caractérisation utilisés par les producteurs étaient la précocité, la couleur des racèmes, la taille de la graine, la taille des plants et la productivité (Tableau 4)

Le critère le plus important était la durée du cycle des cultivars pour laquelle les cinq cultivars ont été regroupés en trois groupes phénologiques :

- un cultivar à cycle court, extra précoce : "Takotapuaka" qui veut dire «fonio de poule ». Il est traduit littéralement comme « fonio qu'on récolte vite ». Son cycle dure 2,5 à 3 mois et il est appelé de ce fait variété de soudure. Il est semé à mi-mai et récolté en août. Les tiges sont de petite taille. Les racèmes, en nombre de 2 à 4 par panicule, sont blancs à la floraison et prennent une couleur grise à la maturité. Les grains sont de petites tailles et ont une couleur grise (Tableau $4)$.

- Deux cultivars à cycle moyen : "Iporapia" et "Iporwan". Ils sont semés en mi- 
mai et récoltés en septembre. Leur cycle varie entre 3,5 et 4 mois : "Iporapia"signifie « fonio précoce blanc» en Ditamari. Son nom générique peut être traduit comme «fonio rapide ». Son cycle dure environ 3 mois et demi. Les racèmes, en nombre de 2 à 4 , sont blancs à la floraison et un peu blanchâtre à la maturité Les grains sont de petites tailles et ont une couleur blanchâtre à la maturité (Tableau 4). "Ipoowan"signifie «fonio rouge » en Ditamari. Son cycle dure environ 4 mois. Les tiges sont élancées. Les racèmes, en nombre de 2 à 5 par panicule sont longs. ils sont de couleur rougeâtre à la floraison et tendent au brun à la maturité. Les grains sont de grandes tailles et ont une couleur rouge foncée ou brune foncée à maturité. C'est une variété assez productive quand elle est cultivée près des bas-fonds.

- Deux cultivars à cycle long : "Ipuaka-ini" et "Ipuaka-ida". Leur cycle de production dure environ 5 mois. Ils sont semés en mi-mai (avec la perturbation due aux pluies, celle-ci peut varier) et récoltés en octobre. Les tiges sont élancées. Les racèmes, en nombre de 2 à 5 par panicule sont longs. Ils sont de couleur blanchâtre à la floraison et tendent au brun à la maturité. Ces cultivars produisent de gros grains, avec une couleur brune à la maturité. Ils sont assez productifs.

\section{Mode d'accès aux semences et conservation des cultivars}

Des producteurs enquêtés, 98,57\% (138 sur 140) ont obtenu leur semence par prélèvement sur la récolte précédente. C'était pour la plupart des semences héritées de leurs ancêtres et qui sont transmises de génération en génération. Les deux producteurs restant avaient obtenu leurs semences, l'un par échange, l'autre par achat. La conservation de ces semences se fait in-situ, avec un renouvellement annuel. $\mathrm{La}$ majorité des producteurs enquêtés $(94,29 \%)$ sont produit un seul cultivar à la fois (132 sur 140). Seulement $1,43 \%$ (2 sur 140 ) en avaient produit deux à la fois, tandis que 4,29\% (6 sur 140) n'avaient pas cultivé le fonio en 2013.

\section{Distribution géographique des cultivars à Boukoumbé}

"Iporapia" et "iporwan" sont les cultivars les plus répandus à Boukoumbé : Ils étaient cultivés presque partout dans la commune, et dans chaque arrondissement par un grand nombre de producteurs enquêtés pour "Iporapia" (Tableau 5). Les cultivars
"Takotapuaka" et "Ipuaka-Ida" sont moins répandus : ils étaient produits chacun dans trois (3) arrondissements sur sept (7).

"Ipuaka-Ini" et "Ipuaka-Ida" sont très peu cultivés : ils sont produits dans chaque arrondissement par très peu de producteurs enquêtés.

Le maximum de diversité variétale a été observée dans les villages de Boukoumbé centre et Natta avec pour chacun quatre (4) des cinq (5) cultivars recensés. Le village de Manta a enregistré la plus faible diversité avec seulement deux des cinq (5) cultivars recensés (Tableau 5).

\section{Distribution ethnique des cultivars à Boukoumbé \\ De l'échantillon enquêté, il ressort que} les Ditamaris ont produit tous les cultivars, mais préférentiellement "Iporapia" (50,88\%), "Iporwan" (23,68\%) et "Takotapuaka" $(12,28 \%)$. Les Lamba avaient cultivé seulement "Takotapuaka" $(63,64 \%)$ et "Iporapia" (36,36\%). Les M'bermè avaient produit presque tous les cultivars à l'exception de "Ipuaka-Ida", mais avec une légère préférence pour "Takotapuaka" (Tableau 6).

Les cultivars "Iporapia" et "Takotapuaka" ont été pratiqués par toutes les ethnies."Iporapia", "Iporawan" et "Takotapuaka" ont été par ordre d'importance les cultivars les plus produits (Tableau 6).

\section{DISCUSSION}

A l'image des grandes zones de production du fonio au Togo, les producteurs de fonio à Boukoumbé sont en majorité des analphabètes, des animistes et très peu sont des femmes (Adoukonou-Sagbadja et al., 2006). Mais à la différence du Togo, on a dénombré au Bénin beaucoup de jeunes adultes (20 à 30 ans), et très peu de personnes âgées de plus de cinquante (50) ans.

L'étude des cultivars rencontrés montre qu'en 10 ans, la diversité variétale n'a pas diminué. En effet, d'après les descriptions, les cultivars identifiés en 2013 correspondent assez bien (aux complexités d'appellations près) aux cinq morphotypes décrits en 2003 par Dansi et al. (2010). Ce résultat suggère que la conservation in situ en cours dans la commune de Boukoumbé a préservé jusque-là la variabilité génétique des accessions de fonio. Cela pourrait en partie s'expliquer par les rôles socioculturels joués par ces cultivars dans les rites traditionnels d'une population en majorité animiste (Gadou Dakouri, 2001; 
Akpavi et al., 2013). La majorité des producteurs maintiennent une seule variété à la fois. La majorité hérite également les cultivars de leurs ancêtres et prélèvent leurs semences sur les récoltes précédentes. Des comportements similaires ont été rapportés au Togo par Adoukonou-Sagbadja et al. (2006). Ce mode de gestion in-situ préserve assez bien l'identité génétique de ces cultivars en limitant les risques de mélange liés à l'exploitation simultanée de plusieurs cultivars de si petites tailles de graines. Toutefois, les cultivars de précocité extrême (le type très précoce "Takotapuaka" et les tardifs "Ipuaka-ini" et "Ipuaka-ida") ont été les moins cultivés, et par conséquent, les plus menacés de disparition. En effet, les cultivars très précoces arrivant à maturité en période de pluies posent aux producteurs des problèmes de séchage et sont par conséquent sujets à des pourritures de récoltes. Ce serait le cas par exemple de "Takotapuaka"(Yoro) qui est désormais rare (voire inexistant) à Boukoumbé-centre, Manta, Natta et Koussoucouingou. Les cultivars trop tardifs quant à eux, n'arrivent pas à toujours boucler leur cycle dans de bonnes conditions hydriques et seraient par conséquent incertains du point de vue du rendement. Ainsi, les cultivars de longueurs de cycle extrêmes (les extra précoces et les très tardifs) étant ainsi très peu préférés par les agriculteurs, sont menacées de disparition à terme, si aucune mesure n'est prise pour les sauvegarder.

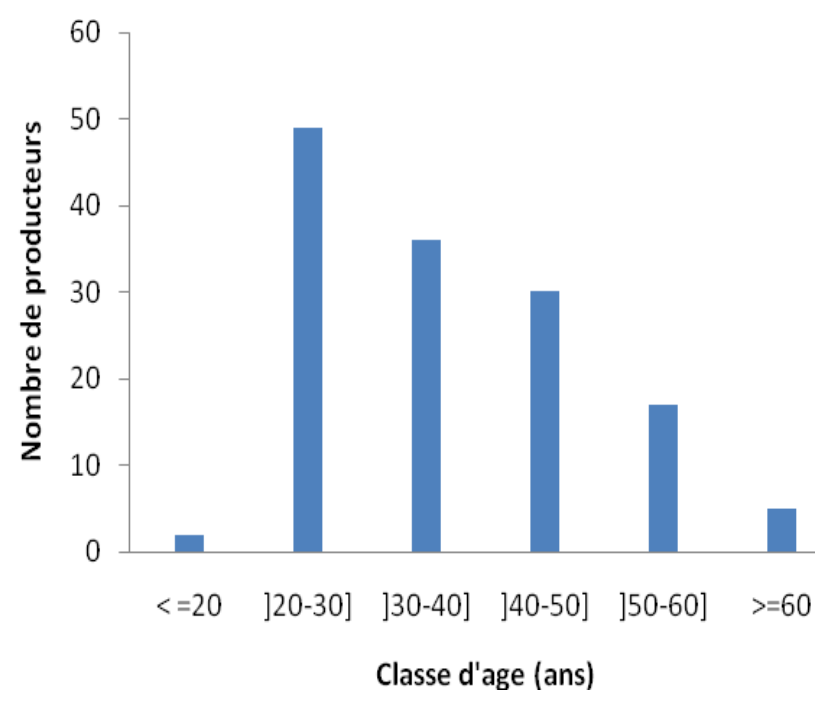

Figure 2 : Répartition par classe d'âge des producteurs enquêtés.

Tableau 1 : Localités couvertes et répartition des producteurs enquêtés individuellement par localité (entre parenthèse).

\begin{tabular}{lccc}
\hline Arrondissements & \multicolumn{3}{c}{ Villages } \\
\hline Dipoli & Dikoumini (6) & Dissapolie (6) & Otanongou (5) \\
Korontière & Tassayota (6) & Entré korontière (5) & Koucongou (7) \\
Tabota & Yatier (8) & Tabota (7) & Koubintiégou (7) \\
Boucoumbé-centre & Ditchindia (13) & kouna_dorgou (10) & Kousétiégou (9) \\
Kousoucoingou & Koussoucoingou-centre (5) & Koukouankouangou (3) & Koukouatchingou (5) \\
Manta & Diporko-1 (8) & Dimatima (9) & \\
Natta & Koudorgou (7) & Kounagou (6) & Dipokorfontri (8) \\
\hline Les nombre entre parenthèse correspondent au nombre de producteurs enquêtés.
\end{tabular}


Tableau 2: Diversité des appellations de fonio recensées à Boukoumbé en 2013.

\begin{tabular}{|c|c|c|c|c|c|c|c|}
\hline Arrondissement & Village & Ditamari & Autres noms & M'bermè & Autres noms & Lamba & Autres noms \\
\hline \multirow{9}{*}{ Dipoli } & \multirow{4}{*}{$\begin{array}{l}\text { Mantcharie } \\
\text { (Lamba) }\end{array}$} & Dakotapora; & - & Ikantoni & - & Yoro & - \\
\hline & & Ipordapia & - & Ipooyè & Ipoodapè & Afféséhumlum & Afféhumlum \\
\hline & & Ipordawan & - & Ipoognirmè & - & Affétasiame & Affésiamon \\
\hline & & Tig-pété & - & Ig-piéri & - & Hôlôm* & Folom; Fouloum \\
\hline & \multirow{5}{*}{$\begin{array}{l}\text { Dissapolie } \\
\text { (M'bermè) }\end{array}$} & Datépora & Dakotapora; & Ikantoni & - & Yoro & - \\
\hline & & Ipordawan & - & Ipoognirmè & - & Affésiamon & - \\
\hline & & Ipordapia & - & Fékpatinanfè & Ipooyè & afféhumlum & - \\
\hline & & Tig-pété & - & Ignonpiéri* & - & Holom & - \\
\hline & & Iporni & - & Dipoomondé* & Ipoomondé & Tamaou & - \\
\hline \multirow{4}{*}{ Tabota } & \multirow{4}{*}{$\begin{array}{c}\text { Yatier } \\
\text { (Ditamari) }\end{array}$} & Ikantoni* & Ikantoga; Kotapoada & Ikantoni & - & Yoro & - \\
\hline & & Nam'ba & Ipordapia & kpatinanfè & - & Afféhumlum & - \\
\hline & & ipordawan & - & Ipoognirmè & - & Affésiamon & - \\
\hline & & Ignonpéré & $\begin{array}{c}\text { Tig-pété; } \\
\text { Koupornigou }\end{array}$ & Ig-piéri & - & Holom & - \\
\hline \multirow{4}{*}{ Manta } & \multirow{4}{*}{$\begin{array}{l}\text { Diporko-1 } \\
\text { (Ditamari) }\end{array}$} & Tintingoula* & - & Ikantoni & - & Yoro & - \\
\hline & & Ipordapia & Koupordagou; Iporpia & Ipooyè & - & afféhumlum & - \\
\hline & & Ipordawan & Iporwan; Iporawan & Ipoognirmè & - & Affésiamon & - \\
\hline & & Iporni & Ini & Ipoomondé & - & tamaou & Tamao \\
\hline \multirow{2}{*}{ Boukombé-Centre } & \multirow{2}{*}{$\begin{array}{l}\text { vers nadoba } \\
\text { (Ditamari) }\end{array}$} & Ipuaka-ini & Iporni; Ini & Ipoomondé & - & tamaou & - \\
\hline & & Ipuaka-ida & Koupordagou & Ipooyè & Iporapia & afféhumlum & - \\
\hline
\end{tabular}


E. SEKLOKA et al. / Int. J. Biol. Chem. Sci. 9(5): 2446-2458, 2015

\begin{tabular}{|c|c|c|c|c|c|c|c|}
\hline & & Ipordawan & Iporawan & Ipoognirmè & - & Affésiamon & - \\
\hline & & Datépora & Kotapoaga & Ikantoni & - & Yoro & - \\
\hline & \multirow{4}{*}{$\begin{array}{c}\text { vers natta } \\
\text { (Ditamari) }\end{array}$} & Iporahouwan & $\begin{array}{l}\text { Ipuagahouin; Iporwan; } \\
\text { Iporawan }\end{array}$ & Ipoognirmè & - & Affésiamon & - \\
\hline & & tententra & Ipordapia & Ipooyè & kpatinanfè & afféhumlum & - \\
\hline & & Ipordagou & Koupordagou & - & - & - & - \\
\hline & & Iporni* & Ini; Ipornouri & Ipoomondé & - & tamaou & - \\
\hline \multirow{10}{*}{ Natta } & \multirow{5}{*}{$\begin{array}{l}\text { koudorgou } \\
\text { (Ditamari) }\end{array}$} & Ipordapia & $\begin{array}{l}\text { Koupordapègou; } \\
\text { tententra;Iporapia }\end{array}$ & Ipooyè & kpatinanfè & afféhumlum & - \\
\hline & & Ipordawan & Iporawan & Ipoognirmè & - & Affésiamon & - \\
\hline & & Koupordagou & Ipordagou & - & - & - & - \\
\hline & & Iporsoukouanta* & $\begin{array}{l}\text { Laporssoukouanta; } \\
\text { tig-pété }\end{array}$ & Ig-piéri & Ig-péri & Holom & - \\
\hline & & Ipornouri* & Iporni & Ipoomondé & - & tamaou & - \\
\hline & & Tententira & dakotapora & Ikantoni & - & Yoro & - \\
\hline & & Inayantapuaka & ipoopia & Ipooyè & kpatinanfè & afféhumlum & - \\
\hline & koudorgou & Koupoodaaku & - & - & - & - & - \\
\hline & (Ditamarı) & Ipoodanuri & Ipooni* & Ipoomondé & - & tamaou & - \\
\hline & & Ipoodawua & Iporawan & Ipoognirmè & - & Affésiamon & - \\
\hline & & takotapora & Kotapora; Datépora & Ikantoni & - & Yoro & - \\
\hline & & Tig-pété & - & Ignonpété* & Tig-pété & Holom & - \\
\hline Korrontiere & (M'bermè) & Iporni & Koupornigou & Ipoomondé* & Dipooakouani; Ipooni & Tamaou & - \\
\hline & & Ipordawan & Monpoognirmè & Ipoognirmè & - & Affétasiame & Affésiamon \\
\hline
\end{tabular}


E. SEKLOKA et al. / Int. J. Biol. Chem. Sci. 9(5): 2446-2458, 2015

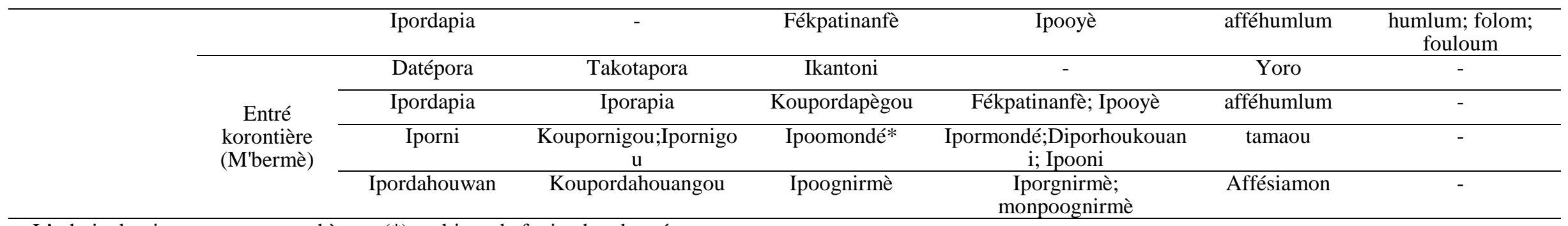

L'ethnie dominante entre parenthèses ; (*): cultivar de fonio abandonné.

Tableau 3 : Cultivars de fonio produits à Boukoumbé et appellations chez les diverses ethnies.

\begin{tabular}{|c|c|c|c|}
\hline Cultivar & Ditamari (Otamari) & M'bermè (Yindé) & Lamba \\
\hline Iporapia & $\begin{array}{l}\text { Iporpia, Ipia, Iporapia, Ipordapia, Namba, Koupordagou, } \\
\text { Ipoopia, Tententra, Koupordapègou, Inayantapuaka }\end{array}$ & $\begin{array}{c}\text { Fékpatinanfè, Ipooyè, Ipoodapè, Kpatinanfè, } \\
\text { Koupordapègou }\end{array}$ & $\begin{array}{l}\text { Afféhumlum, humlum, } \\
\text { Folom, fouloum }\end{array}$ \\
\hline Iporawan & $\begin{array}{l}\text { Iporwan, Ipordawan, Iporawan, Ipuagahouin, } \\
\text { Iporahouwan, Ipoodawua, Monpoognirmè, } \\
\text { Ipordahouwan, Koupordahouangou }\end{array}$ & Ipoognirmè, Ipormondé, Iporgnirmè, monpoognirmè & Affésiamon, Affétasiame \\
\hline Takotapuaka & $\begin{array}{c}\text { Takotapuaka, Dakotapora, Datépora, Ikantoni, Ikantoga, } \\
\text { Kotapoada, Kotapoaga, Tintingoula, Tententira }\end{array}$ & Ikantoni & Yoro \\
\hline Ipuaka-Ini & $\begin{array}{c}\text { Ipuaka-Ini, Iporni, Ini, Ipornouri, Ipooni, Ipoodanuri, } \\
\text { Koupornigou, Ipornigou }\end{array}$ & $\begin{array}{l}\text { Dipoomondé, Ipoomondé, Dipooakouani, Ipormondé, } \\
\text { Diporhoukouani, Ipooni }\end{array}$ & Tamaou, Tamao \\
\hline Ipuaka-Ida & $\begin{array}{c}\text { Ipuaka-Ida, Tignonpété, Koupornigou, Ignonpéré, } \\
\text { Koupordagou, Ipordagou, Iporsoukouanta, } \\
\text { Laporssoukouanta }\end{array}$ & Ignonpiéri, Tignonpété, Ignonpété, Holom, & $\begin{array}{l}\text { Holom, Folom, Fouloum, } \\
\text { Ignonpiéri, }\end{array}$ \\
\hline
\end{tabular}


Tableau 4: Morphologie et production des cultivars (décrites par les producteurs).

\begin{tabular}{|c|c|c|c|c|c|}
\hline Paramètres observés & Takotapuaka & Iporapia & Iporwan & Ipuaka-ini & Ipuaka-ida \\
\hline Précocité & Extra-précoce (2,5 à 3 mois) & Moyen (3,5 à 4 mois) & Moyen (3,5 à 4 mois) & Tardif (environ 5 mois) & Tardif environ 5 mois) \\
\hline Taille des plants & Petite & Petite à moyenne & Elancé & Elancé & Elancé \\
\hline Nombre de racèmes par panicule & 2 à 4 & 2 à 4 & 2 à 5 & 2 à 5 & 2 à 5 \\
\hline Longueur des racèmes & Court & Moyen & Long & Long & Long \\
\hline Couleur des racèmes à la floraison & Blanc & Blanc & Rougeâtre & & \\
\hline Taille de la graine & Petite & Petite & Moyenne & Gros & Gros \\
\hline Couleur de la graine & Grise & Blanchâtre & brune & Brune & Brune \\
\hline Production & Faible & Moyenne & $\begin{array}{l}\text { Bonne si cultivée près des } \\
\text { bas-fonds }\end{array}$ & Bonne & Bonne \\
\hline
\end{tabular}

Tableau 5: Nombre de producteurs enquêtés par cultivars et par arrondissement.

\begin{tabular}{lcccccc}
\hline Arrondissement & Iporapia & Iporawan & Takotapuaka & Ipuaka-Ini & Ipuaka-Ida & Total \\
\hline Dipoli & 5 & 3 & 7 & - & - & 15 \\
Korontière & 10 & - & 4 & 2 & - & 16 \\
Tabota & - & 7 & 14 & - & 1 & 22 \\
Boukoumbé-centre & 14 & 13 & - & 3 & 2 & 32 \\
Kousoucoingou & 10 & 2 & - & 1 & - & 13 \\
Manta & 15 & - & - & 2 & - & 17 \\
Natta & 10 & 5 & - & 2 & 4 & 21 \\
\hline Total & 64 & 30 & 25 & 10 & 136 \\
\hline Six des producteurs enquêtés n'ont pas cultivé le fonio ; mais le total fait ici 136 au lieu de 134 producteurs parce que deux d'entre ceux
\end{tabular}

Six des producteurs enquêtés n'ont pas cultivé le fonio ; mais le total fait ici
qui en ont cultivé ont produit simultanément deux variétés à la fois chacun. 
Tableau 6: Nombre de producteurs enquêtés par cultivars et par ethnie.

\begin{tabular}{lcccccc}
\hline Ethnies & Iporapia & Iporawan & Takotapuaka & Ipuaka-Ini & Ipuaka-Ida & Total \\
\hline Ditamari & 58 & 27 & 14 & 8 & 7 & 114 \\
lamba & 4 & & 7 & & & 11 \\
M'bermè & 2 & 3 & 4 & 2 & 11 \\
\hline Total & 64 & 30 & 25 & 10 & 7 & 136 \\
\hline \multicolumn{5}{l}{ Six des producteurs enquêtés n'ont pas cultivé le fonio ; mais le total fait ici 136 au lieu de 134 producteurs } \\
parce que deux d'entre ceux qui en ont cultivé ont produit simultanément deux variétés à la fois chacun.
\end{tabular}

Ces résultats sont en harmonie avec ceux trouvés par Dansi et al. (2010) qui ont montré que ces cultivars étaient de plus en plus produits par peu de ménages sur de toutes petites superficies. Les cultivars "Iporapia" et "Iporwan", à cycle intermédiaire sont les plus répandus à Boukoumbé.

La comparaison de différentes études d'identification des cultivars de fonio est fastidieuse et compliquée par la diversité et la complexité de noms vernaculaires utilisés pour désigner ces cultivars dans la zone d'étude. Les présents travaux ont permis de recenser par cultivar les différentes appellations dans les différentes langues pratiquées et ceci dans les différentes communes prospectées. Toutefois, même si cette étude comme celle de Dansi et al. (2010) confirment globalement que cinq(5) cultivars sont pratiqués dans la zone dont quatre seraient du Digitaliaexilis et un du Digitalia iburua selon Dansi et al. (2010), deux différences d'appellation importantes méritent d'être signalées. Premièrement, "Ipoaga" (nom attribué au seul cultivar D. iburua par Dansi et al., 2010) n'est que le nom générique du fonio dans la zone. "Ipoaga" ne correspond donc pas à un cultivar particulier. Deuxièmement, une inversion d'appellation est apparue avec Dansi et al. (2010) en ce qui concerne le cultivar "Ipuaka-ini". En effet, à la différence des travaux de Dansi et al. (2010), le cultivar "Ipuaka-ini" (encore appelé "Iporni") correspondrait plutôt à "Tamaou" en Lamba et non à "Folom". Cette différence est d'autant plus importante que le cultivar "Tamaou" a été décrit par Dansi et al. (2010) comme étant la seule espèce de $D$. iburua cultivée dans la zone.

La plus grande richesse variétale a été mise en évidence à Boukoumbé-centre et à Natta. Cette diversité variétale a été fondamentalement entretenue par les Ditamari et dans une moindre mesure par les M'bermè. Ces résultats, comme ceux de Dansi et al.
(2010) ont confirmé que les producteurs ont une bonne connaissance de leur matériel végétal et qu'en termes de classification, d'identification et d'utilisation, leurs connaissances sont d'une extrême importance pour les sélectionneurs dans l'amélioration génétique de cette culture.

\section{Conclusion}

La diversité variétale décrite en 2003 , soit dix (10) ans plus tôt, dans la zone de Boukoumbé et ses environs a été maintenue à travers le mode de conservation traditionnel utilisé par les agriculteurs. Toutefois, l'étude a confirmé que les cultivars de précocité extrême (très précoces et très tardifs) sont de moins en moins cultivés, et sont par conséquent menacées de disparition, ce qui appelle des actions urgentes pour la préservation ex situ de ces cultivars. Ce travail a en outre permis d'établir une liste exhaustive des appellations de fonio dans la commune de Boukoumbé qui pourra servir aux études ultérieures d'amélioration variétale de cette culture.

\section{CONFLITS D'INTERETS} d'intérêt.

Les auteurs ne déclarent aucun conflit

\section{CONTRIBUTIONS DES AUTEURS}

ES est l'initiateur de l'étude. Il a participé à toutes les étapes depuis la conception du protocole jusqu'à la rédaction, la soumission et la révision du manuscrit. HA$\mathrm{S}$ a participé à la rédaction du protocole, au suivi des enquêtes de terrain, à l'accompagnement bibliographique et l'amendement du manuscrit. AAP a participé au suivi des enquêtes de terrain, à l'accompagnement bibliographique et à la rédaction du manuscrit. BKY a participé à la réalisation des enquêtes, au dépouillement et à la synthèse des résultats ainsi qu'à la rédaction du manuscrit. F-XB a participé au suivi des 
enquêtes de terrain, au dépouillement, à la synthèse des résultats ainsi qu'à la rédaction du manuscrit. NZ-W a participé à la correction du protocole, au suivi des enquêtes de terrain, au dépouillement, à la synthèse des résultats ainsi qu'à la rédaction du manuscrit.

\section{REMERCIEMENTS}

Nous remercions tous les braves producteurs enquêtés ainsi que les agents du Secteur Communal de Développement Agricole (SCDA) de Boukoumbé pour leur franche et cordiale collaboration.

\section{REFERENCES}

Adoukonou-Sagbadja H, Dansi A, Vodouhè R, Akpagana K. 2006. Indigenous knowledge and traditional conservation of fonio millet (Digitariaexilis Stapf, Digitaria iburua Stapf) in Togo. Biodiversity and Conservation, 15: 2379-2395. DOI: $10.1007 / \mathrm{s} 10531-004-$ 2938-3.

Adoukonou-Sagbadja H, Wagner C, Dansi A, Ahlemeyer J, Daïnou O, Akpagana K, Ordon F, Friedt W. 2007. Genetic diversity and population differentiation of traditional fonio millet (Digitaria spp.) landraces from different agroecological zones of West Africa. Theoretical and Applied Genetics, 115: 917-931. DOI: 10.1007/s00122-0070618-x.

Akpavi S, Kanda M, Odah K, Akpakpah KE, Kossi-Ttrikou K, Boutaré I, Akpagana K. 2013. Valeur socio-culturelle des plantesalimentaires: unfacteur de preservation. European Scientific Journal, 9(32): 383-395.

Apostolidis T. 2005. Représentations sociales et triangulation: enjeux théoricométhodologiques. In Méthodes d'étude des représentations sociales, ERES (ed)"Hors collection": 13-35.

Ballogoun VY, Sagbo FS, Soumanou MM, Toukourou F, Hounhouigan JD. 2012.
Evaluation de la qualité de quelques produits dérivés de deux écotypes de fonio cultivés (Digitariaexilis) au Bénin. Bulletin de la Recherche Agronomique du Bénin (BRAB), 72 : 27-35.

Ballogoun VY. 2013. Systèmes post-récolte, transformation, qualité du fonio et produits dérivés au nord du Bénin. Thèse de Doctorat, Faculté des Sciences Agronomiques, Université d'AbomeyCalavi, p. 152.

CIRAD (Centre de coopération internationale en recherche agronomique pour le développement). 2010. Rapport de l'atelier international : De la connaissance à la valorisation du fonio, Niamey, Niger, du 09 décembre 2010 au 11 décembre 2010,http://fonio.cirad.fr/en/content/dow nload/2337/17675/file/De\%201a\%20con naissance $\% 20 \% \mathrm{C} 3 \% \mathrm{~A} 0 \% 201 \mathrm{a} \% 20$ valori sation\%20du\%20fonio.pdf(consulté le 17 Mai 2015).

Cruz JF, Béavogui F. 2011. Le Fonio, une Céréale Africaine. Quae, CTA, Presses agronomiques de Gembloux.

Dansi A, Adoukonou-Sagbadja H, Vodouhè R. 2010. Diversity, conservation and related wild species of fonio millet (Digitaria spp.) in the northwest of Benin. Gent. Resour. Crop.Evol., 57: 827-839. DOI : 10.1007/s10722-0099522-3.

GadouDakouri M. 2001. La préservation de la biodiversité : les réponses de la tradition religieuse africaine. The African Anthropologist, 8(2): 178-199.

Olivieri I, Vitalis, R. 2001. La biologie des extinctions. Med Sci (Paris), 17(1) : 6369. DOI : $10.4267 / 10608 / 1788$.

Paraïso AA, Sossou ACG, Yegbemey RN, Biaou G. 2011. Analyse de la rentabilité de la production du fonio (Digitariaexilis S.) dans la commune de Boukoumbé au Bénin. J. Rech. Sci. Univ. Lomé (Togo), Série A, 13(1) : 27-37. 